CAHIERS DE

NARRATOLOGIE

\section{Cahiers de Narratologie}

Analyse et théorie narratives

$10.1 \mid 2001$

La voix narrative

\title{
Instance de l'auteur et narration chez Berceo : cohérence de la voix narrative
}

\section{Olivier Biaggini}

\section{(2) OpenEdition \\ 1 Journals}

\section{Electronic version}

URL: http://journals.openedition.org/narratologie/6938

DOI: 10.4000/narratologie.6938

ISSN: 1765-307X

\section{Publisher}

LIRCES

\section{Printed version}

Date of publication: 1 January 2001

Number of pages: 159-170

ISBN: 2914561032

ISSN: 0993-8516

\section{Electronic reference}

Olivier Biaggini, "Instance de l'auteur et narration chez Berceo : cohérence de la voix narrative", Cahiers de Narratologie [Online], 10.1 | 2001, Online since 23 October 2014, connection on 23 February 2021. URL: http://journals.openedition.org/narratologie/6938; DOI: https://doi.org/10.4000/narratologie. 6938 


\title{
INSTANCE DE L'AUTEUR ET NARRATION CHEZ BERCEO : COHÉRENCE DE LA VOIX NARRATIVE
}

\author{
Olivier BIAGGINI \\ Université Paris III
}

Gonzalo de Berceo est l'auteur, au XIIIe siècle, d'une innovation remarquable : il est le premier écrivain castillan connu à avoir intégré son nom à la matière de ses œuvres. Cette signature personnelle a évidemment des conséquences sur le statut qu'il accorde à son écriture et sur l'image qu'il forge de sa propre fonction d'auteur. Par ailleurs, dans ses poèmes, se manifeste à la première personne du singulier une instance qui déclare assumer la production de l'œuvre et de la matière narrative qui la constitue. À cet égard, la première strophe des Milagros est emblématique' ${ }^{1}$ :

Amigos e vassallos de Dios omnipotent,

si vós me escuchássedes por vuestro consiment, querríavos contar un buen aveniment : terrédeslo en cabo por bueno verament.

Amis et vassaux de Dieu tout puissant, si vous aviez l'obligeance de m'écouter, je voudrais vous conter un bon événement : à la fin, vraiment, vous le trouverez bon.

La voix mise en scène par le texte écrit n'est soumise à aucune identification dans certaines œuvres (traités liturgiques ou mariologiques) mais, dans les poèmes proprement hagiographiques, elle est rapportée au nom de Gonzalo de $\mathrm{Berceo}^{2}$. Or, ces poèmes qui portent la mention du nom de

Je cite les œuvres de Berceo d'après Obra completa (coord. Isabel Uría, Madrid : Espasa Calpe, 1992).

2 Le nom de l'auteur figure dans les dernières strophes de San Millán (489), Santo Domingo (757 et 775) et Santa Oria (205, d'après Isabel Uría). Les Milagros, quant à eux, le mentionnent dès la 
l'auteur sont également ceux qui se caractérisent, de la façon la plus immédiate, par une forte dimension narrative.

Ce constat nous conduit à nous interroger sur la nature de cette instance que le nom de Gonzalo de Berceo est censé venir identifier : est-ce un auteur ou un narrateur ? Pour une œuvre moderne, cette question n'aurait peut-être pas de véritable portée : l'instance de l'auteur est première, issue de l'existence en chair et en os d'une personne qui écrit et qui, en principe, appose un nom au seuil de l'œuvre. Elle se trouve relayée par l'instance seconde du narrateur dès que le récit commence. Ce je qui prétend raconter serait donc celui d'un narrateur. Qu'il se nomme lui-même Gonzalo de Berceo n'y change rien : le narrateur revendique la même identité que l'auteur mais les deux instances demeurent séparées. Même dans le cas d'un récit anonyme, nos conventions modernes posent l'auteur comme instance première. Inséparable d'une telle conception, se tient la notion de propriété littéraire, soit l'idée qu'un texte se rapporte à une personne dans une relation exclusive de responsabilité. Or, cette notion est à peu près étrangère à la mentalité médiévale. Si l'anonymat est accidentel pour une œuvre moderne, il ne l'est que partiellement pour une œuvre médiévale parce que l'instance de l'auteur ne saurait être perçue comme une instance première dans le processus de production de l'œuvre. Le Moyen Âge classique, par le terme latin d'auctor ou ses équivalents vernaculaires, ne désigne pas un écrivain contemporain mais réserve cette appellation aux auteurs anciens, sacrés ou profanes, investis d'une certaine autorité. ${ }^{3}$ Un écrivain médiéval ne se considère presque jamais lui-même comme un auctor.

Chez Berceo, l'instance de l'auteur est un désir, une figure idéale du producteur du discours : le poète ne saurait la revendiquer de façon directe. L'hypothèse de mon travail est que l'auteur a besoin des récits et de leur pouvoir énonciatif

deuxième strophe. Le Martirio de San Lorenzo n'offre aucune signature d'auteur mais ce poème nous est parvenu dans une version incomplète, soit que Berceo ne l'ait pas achevé, soit que la fin du poème ait été perdue.

${ }^{3}$ Cf. Gérard LECLERC, Histoire de l'autorité, (Paris : PUF, 1996), p. 99-135. 
pour parvenir à se construire. La voix narrative qui gouverne les récits de Berceo, apparemment fragile et aliénée, apparaît peut-être comme l'un des principaux lieux où se constitue une instance de l'auteur cohérente. Pour savoir qui est auteur, il faut d'abord savoir qui raconte. J'examinerai, en premier lieu, le rapport de la voix narrative de Berceo à celle de ses sources latines; et en second lieu, le rôle joué par d'autres voix, de nature sainte ou divine, qui interagissent avec les deux précédentes pour produire le récit.

\section{Berceo et la voix de la source}

Tous les récits de Berceo sont ponctués de références à des textes latins antérieurs. Des éléments du récit sont ainsi attestés, avec plus ou moins de conviction, par des formules telles que "dizlo la escriptura » ou " como diz la leyenda », dans lesquelles le texte cité peut être explicitement identifié ou non. A cet égard, l'on doit distinguer des références ponctuelles à des textes scripturaires et des références à la source latine dont le texte roman est une adaptation. Dans ce dernier cas, en effet, la mention d'un écrit antérieur n'est pas seulement un rouage argumentatif destiné à avérer une affirmation, mais révèle aussi que la source est censée offrir la version authentique du récit dans son ensemble. Ce que raconte Berceo en roman et en vers a déjà été raconté en latin et en prose par une source qui est l'origine et la garantie de toute la matière narrative. Ce phénomène tend à brouiller l'identité de la voix narrative. Berceo répète à l'envi que son récit n'a de validité à s'énoncer que dans le sillage d'une énonciation première, investie d'une autorité. De même qu'il ne saurait se définir d'emblée comme auctor, ses récits et la voix narrative qui les produit ne peuvent prétendre immédiatement à une autonomie. Il importe de souligner que cette dépendance ne lie pas seulement deux énoncés mais bien deux énonciations, pas seulement deux récits mais bien deux voix narratives. Au début de San Millán, quand Berceo s'apprête à relater la vie du saint, il présente sa narration comme une lecture :

Qui la vida quisiere de sant Millán saber, e de la su istoria bien certano seer, meta mientes en esto que yo quiero leer 
Celui qui veut savoir la vie de saint Émilien et connaître son histoire en toute certitude, qu'il prête attention à ce que je veux lire. (1abc)

Selon la convention narrative posée, Berceo s'apprêterait à lire le récit tel qu'il apparaît dans la source latine et son œuvre serait cette lecture même. Il est vrai que le verbe leer peut être également interprété dans le sens de la lectio médiévale, c'est-à-dire du commentaire linéaire : leer, ce serait donc aussi bien lire la source que lui adjoindre une interprétation. Au-delà de son sens proprement exégétique, l'interprétation pourrait désigner ici la performance vocale, comme lorsque l'on dit que l'on interprète un chant pour signifier que l'on prête sa voix à une œuvre déjà écrite. En ce sens, la voix narrative se présente elle-même comme l'instrument qui actualise, par une performance prétendument orale, un récit déjà constitué, déjà institué par la source latine. Même lorsqu'une comparaison philologique du récit castillan à sa source met en évidence des innovations, Berceo a coutume de s'en remettre à l'autorité de ce qui a déjà été écrit et raconté. Pourtant, il ne faudrait pas comprendre cette dépendance affichée comme une servilité. San Millán, au terme du récit de la vie et des miracles du saint, mentionne le nom de Berceo et le désigne sans appel comme le seul responsable de l'œuvre :

Gonzalvo fue so nomne qui fizo est' tractado, en Sant Millán de Suso fue de niñez crïado ; natural de Verceo ond' sant Millán fue nado, Dios guarde la su alma del poder del Pecado.

Gonzalvo est le nom de qui fit ce traité, à Saint-Émilien-le-Haut, il fut élevé dès l'enfance ; natif de Verceo, là où est né saint Émilien, que Dieu garde son âme du pouvoir du Péché. (489)

L'aveu initial de dépendance fait donc place à une revendication de responsabilité littéraire, et la définition du récit comme lecture à une définition de l'œuvre dans sa facture (leer est remplacé par fazer). Comment justifier ce retournement de la convention narrative ? Tout se passe comme si la voix narrative dépendante avait contribué à construire une instance de l'auteur indépendante, célébrant ainsi un transfert d'autorité de la source latine vers l'œuvre vernaculaire. Parmi 
les procédés qui servent ce transfert, il est un élément du récit apparemment anecdotique : le texte souligne que le saint est natif de Berceo, hameau dont le poète est lui-même originaire et dont il tire son nom. Or, ce détail biographique, absent de la source latine, est totalement imputable au poète, qui le rappelle à dix reprises au cours du récit (strophes $2,19,27,81$, $92,93,99,115,174,489)$. Cette insistance ne peut être innocente : si Berceo tient tant à rappeler que le saint est né à Berceo, c'est pour exploiter le jeu onomastique en termes d'autorité. Non seulement le poète se montre fier d'être né dans le même village que le saint et exhibe ainsi une forme de piété locale, mais le nom propre de Berceo définit indirectement le poète comme origine : Berceo désigne aussi bien l'origine du saint que celle de l'hagiographie. En exploitant systématiquement ce principe analogique, Berceo, à l'issue du récit et par ce récit, est bien devenu l'auteur d'une œuvre.

L'instance qui assume le récit est donc soumise à une tension qui a des conséquences immédiates en termes d'identité : la voix narrative de Berceo laisse entendre constamment les échos d'une voix antérieure, qui est investie d'une autorité plus forte et qui, en même temps, l'aide à construire une autorité propre. Par le jeu de cette tension, il n'est pas étonnant que le système narratif connaisse des glissements qui peuvent ressembler à des incohérences formelles. Le cas le plus célèbre à cet égard est le Poema de Santa Oria, dans lequel, par deux fois, la voix narrative, sans justification explicite, passe de Berceo à Munius, auteur-narrateur de la source latine. Or, Munius n'est pas seulement l'auteur du récit dont Berceo s'inspire, mais aussi un personnage qui, dans l'histoire, recueille les confessions de la sainte et celles de sa mère, Amuña, également affectée de visions ${ }^{4}$. Par ailleurs, la question de la voix est un thème central de l'œuvre, puisque, dans ses visions, Oria est en quête d'un personnage mystérieux appelé Voxmea, qu'elle ne mérite de rencontrer qu'après sa mort. Dans le système de transmission des visions, Munius assume essentiellement une fonction testimoniale :

4 Sur le rôle médiateur de Munius, voir Julian Weiss, "Writing, Sanctity, and Gender in Berceo's Poema de Santa Oria", Hispanic Review, 64-4 (1996), p. 447-465. 
même si, par définition, il ne saurait avoir directement accès à la vision, l'auctoritas qu'il appose au récit vaut pour l'autorité d'un témoignage direct. L'intimité qui le lie à la sainte et à sa mère permet la mise en circulation d'un récit authentique, substitut impeccable de la vision. Tout naturellement, comme rédacteur d'une hagiographie, Munius se pose à la fois comme un antécédent et un double de Berceo. Or, les deux glissements narratifs par lesquels Berceo cesse temporairement d'assumer la narration au profit de Munius interviennent, dans l'histoire, au moment où le personnage affecté par la vision s'apprête à la transmettre à Munius, c'est-à-dire au moment où est sollicitée sa fonction testimoniale. Par une aliénation apparente de sa voix au profit d'un narrateur qui peut raconter en tant que témoin direct, Berceo confère à son récit une authenticité accrue. Le glissement d'une voix à l'autre obéit, en termes narratifs, au même processus qu'un argument d'autorité : il permet de transférer l'autorité du récit de Munius à celui de Berceo. Le système des voix, loin d'être incohérent, est tout entier orienté vers la construction d'une instance de l'auteur investie d'une auctoritas. La démarche est ici à la fois analogique et généalogique. Berceo se pose en héritier du récit authentique de Munius et en tire tout le profit qu'il peut en termes d'auctoritas:

Qui en esto dubdare que nos versificamos, que non es esta cosa tal como nos contamos, pecará duramente en Dios que adoramos, ca nos quanto dezimos escripto lo fallamos.

Celui qui doutera de ce que nous versifions, et dira que la chose n'est pas telle que nous la contons, péchera gravement contre Dieu que nous adorons, car tout ce que nous disons, écrit le trouvons. (6)

Du même coup, ce n'est plus seulement la voix de la source, mais également la voix de la sainte et, au-delà, celle de Dieu que le récit prétend prolonger selon une chaîne de transmission sans faille : la voix divine recherchée par la sainte, cette "Voxmea » introuvable, est providentiellement assimilée à la voix narrative de Berceo. 


\section{Berceo et la voix céleste}

Dans la voix de Berceo résonne également celle des saintes figures que le récit exalte. Selon un principe analogique, il n'est pas rare que le texte associe indirectement la figure du poète à celle du saint, voire à celle de Dieu, en les considérant toutes comme des figures de l'origine et de la production. Certes, dans un premier temps, le saint joue principalement comme une instance de réception plutôt que d'énonciation : alors que dans San Millán, il n'est que personnage du récit, il devient aussi son possible destinataire dans Santo Domingo, puisque Berceo, en échange du service rendu, lui demande, dans les vingt dernières strophes du poème, de lui accorder sa protection et son intercession auprès de Dieu. Cette conception classique de l'hagiographie comme un service qui appelle une récompense de la part du saint trouve une application encore plus nette dans les œuvres mariales, parce que la Vierge, co-rédemptrice de l'humanité, est censée dispenser une intercession plus efficace. Cependant, la sainte figure n'est pas seulement sollicitée comme destinataire de l'œuvre achevée : le poète l'exhorte aussi à participer à l'œuvre en cours, au processus même de la narration. Dans Santa Oria, une même strophe invite la sainte à exercer les fonctions de guide et d'intercesseur :

Luego en el comienço e en la primería, a ella mercet pido, ella sea mi guía ; ruegue a la Gloriosa, Madre Sancta María, que sea nuestra guarda de noche e de día.

Dès le début et en tout premier lieu, je lui demande grâce, qu'elle soit mon guide ; qu'elle prie la Glorieuse, notre Mère Sainte Marie, d'être notre gardienne, de jour comme de nuit. (3)

Dans les œuvres mariales, l'appel à une intervention de la Vierge pour guider le récit devient systématique. Le Duelo de la Virgen commence par une invocation de ce type :

\section{si Ella me guiasse por la gracia divina, querría del su duelo componer una rima.}

si Elle me guidait par la grâce divine, je voudrais de son deuil composer un poème. $(1 \mathrm{~cd})$ 
L'aide de la Vierge est ici sollicitée en vertu de la grâce de Dieu, ce qui montre que l'intervention mariale dans le processus d'élaboration de l'œuvre est elle-même une forme d'intercession. Quant au poème des Loores de Nuestra Señora, il invoque l'aide mariale comme la condition même du bon déroulement des épisodes narratifs :

En tu feduça, Madre, de ti quiero decir cómo vino el mundo Dios por ti redemir ;

Tú m'da bien empeçar, Tú m'da bien acomplir, que pueda tu materia quál o cómo seguir.

Par ta foi, Mère, je parler de toi et dire comment Dieu vint, par toi, racheter le monde ;

donne-moi bon début, donne-moi bonne fin, pour que je sache m'orienter en ta matière. (3)

Les Milagros poussent encore plus loin cette association du personnage au processus d'élaboration littéraire en considérant qu'une telle écriture guidée relève du miracle :

Terrélo por miráculo que lo faz la Gloriosa si guïarme quisiere a mí en esta cosa ;

Je tiendrai cela pour un miracle que fait la Glorieuse si elle daigne me guider dans cette entreprise (46ab).

Dans ce cas, la composition du récit des miracles, grâce à l'intervention de la Vierge, devient un miracle de plus, qui donne à l'œuvre de Berceo une auctoritas sans équivalent dans les autres poèmes. Globalement, ces déclarations posent toutes de façon analogue le problème de l'identité de la voix narrative à l'œuvre dans le texte. Selon les conventions idéales du récit, est-ce Berceo ou la Vierge qui raconte? N'oublions pas que, par ailleurs, le prologue des Milagros allègue que les Évangiles ont été dictés à leurs auteurs humains par la Vierge elle-même (21-22). Selon quelles modalités la voix narrative peut-elle encore se donner le nom de Berceo tout en se réclamant de Marie ?

Cette question est traitée dans le Duelo à partir d'une mise en abyme de la voix narrative. Berceo n'aborde pas directement le récit de la lamentation de Marie mais introduit le personnage de saint Bernard, qui est censé avoir parlé avec la Vierge en vision et avoir recueilli de sa bouche les détails de son histoire et de ses sentiments au pied de la Croix. Il se 
trouve que des textes de saint Bernard sont très probablement la source effective du Duelo. Cependant, cette source est ici convoquée non comme texte écrit mais comme récit et, qui plus est, comme récit intégré à celui de Berceo. La narration de Berceo la traite comme si elle s'exposait en même temps qu'elle. Le narrateur extradiégétique est relayé par une instance de narration intradiégétique investie d'une autorité particulière parce qu'elle retranscrit les paroles et les pensées les plus secrètes de la Vierge. Elle est composée de deux voix, celle de Bernard et celle de la Vierge, qui dialoguent pour élaborer une œuvre. Ainsi, Marie déclare à Bernard :

quiero que compongamos yo e tú una prosa.

je veux que nous composions, toi et moi, un poème. (10)

Le verbe " componer », employé quelques strophes plus haut pour désigner le projet d'écriture de Berceo, est repris ici pour définir les modalités du récit où convergent, sans se détruire, la voix de l'auteur terrestre et celle de l'auteur céleste. Par ailleurs, par redoublement de cette structure, la Vierge, lorsqu'elle assume cette instance narrative pour raconter ses propres tourments, en appelle elle-même à l'aide de Dieu :

Conviene que fablemos, en nuestra privanza, del pleito de mi duelo, de la mi malandanza, cómo sufrí martirio sin gladio e sin lanza, si Dios nos ayudara, fer una remembranza.

Il nous faut nous entretenir, en notre intimité, au sujet de mon deuil et de mon malheur, et de comment je souffris le martyre, sans lance ni glaive, si Dieu nous y aidait, faire une remémoration. (44)

Dans l'exégèse médiévale classique, une double autorité de ce type est caractéristique des œuvres inspirées. Saint Bernard et la Vierge sont présentés comme des doubles de Berceo, puisqu'ils invoquent, comme lui, une inspiration qui garantisse l'authenticité du récit. Par une analogie quelque peu forcée, la réussite de Bernard dans son entreprise, soit la fusion de sa voix avec celle de la Vierge, devient la garantie du projet narratif et littéraire de Berceo, qui peut implicitement passer à son tour pour un auteur inspiré. Et, là encore, le principe analogique se combine à un principe généalogique, puisque Berceo, au bout de la chaîne de transmission, est 
censé recueillir, grâce à saint Bernard, les paroles authentiques de la Vierge, elles-mêmes prononcées avec l'aide de Dieu.

Dans la pratique, malgré une certaine instabilité de la voix, la convention narrative posée trouve une application cohérente. La critique a parfois dénoncé comme des incohérences les glissements d'un narrateur à l'autre qui s'opèrent dans le Duelo. Au moment où la Vierge rapporte les mots qu'elle a échangés avec le Christ agonisant, une voix narrative extérieure - dont on ne sait si elle revient à Bernard ou à Berceo - fait irruption pour différencier les discours (« Recudió el Señor», « Dixo la Madre », etc. $)^{5}$. À l'issue du dialogue, la Vierge perd connaissance si bien qu'elle ne peut logiquement prendre en charge la narration des faits qui suivent. C'est donc la voix non identifiée qui l'assume, en s'appuyant explicitement sur le récit de l'Évangile de Mathieu. Le narrateur indéterminé a donc essentiellement la fonction de compléter le récit des événements sacrés tant que la Vierge ne peut en être le témoin. Il apporte un témoignage subsidiaire, fondé sur l'auctoritas du récit évangélique. Quelque dix strophes plus loin, dès que la Vierge, en tant que personnage de l'histoire, a retrouvé ses esprits, sa voix peut aussi reprendre en charge le récit. Ce phénomène nous paraît relever de ce que Gérard Genette appelle métalepse, soit une interaction transgressive entre le monde de la diégèse et celui du récit ${ }^{6}$ : il apparaît chez Berceo sous bien d'autres formes. Tout cela montre à quel point, dans le Duelo, le système des voix est instable, mais, parce que le poème revendique idéalement le statut d'œuvre inspirée, cette instabilité même est, paradoxalement, source de cohérence. Enfin, ce n'est pas la voix de la Vierge qui achève la narration mais, subrepticement, Berceo reprend la parole sans que l'on puisse déterminer exactement à quel moment se fait la passation de pouvoir d'un narrateur à un autre. La narration est alors couronnée par une invocation finale qui laisse transparaître un formidable désir d'auctoritas : l'instabilité effective du narrateur a

5 Cf. Connie SCARBoRough, "Narrative Voices in Berceo's $E l$ duelo de la Virgen ", Romance Notes, 34-2 (1993), p. 111-118.

${ }^{6}$ Figures III (Paris : Seuil, 1972), p. 243-246. 
permis d'accorder une plus grande stabilité à la figure idéale de l'auteur.

Ainsi, face à la source latine ou à des instances inspiratrices, Berceo, comme narrateur et auteur, compose avec d'autres voix, mais le texte prétend ramener ces interactions à un modèle d'autorité unique et cohérent. En exploitant des figures analogiques et généalogiques de la production du discours, les récits, aussi bien que les arguments théoriques et souvent plus efficacement qu'eux, attribuent à Berceo une aptitude au discours véridique. Nul autre passage que le prologue des Milagros ne donne mieux à voir cette convergence vers la figure désirée de l'auctor. Par l'allégorie du pré marial dont il est à la fois personnage, narrateur et interprète, Berceo nous livre le seul texte de sa main qui s'assume comme fiction, après avoir pris les apparences d'un récit testimonial et autobiographique. C'est dans ce récit fictif que s'incarne le mieux toute la prétention du poète à dire le vrai. La rencontre allégorique du poète et de la grâce mariale se résume dans la figure de Berceo perché dans un arbre du pré, en train d'écrire. Dans l'allégorie, les arbres signifient les miracles de la Vierge, ce qui montre que l'auteur puise son récit à sa source première, au point de le rendre lui-même miraculeux. Par ailleurs, l'acte d'écriture contient toujours implicitement la voix puisque l'arbre du miracle abrite aussi des oiseaux chanteurs qui représentent tous les auteurs vénérables qui ont su louer harmonieusement la Vierge :

Las aves que organan entre essos fructales, que an las dulzes vozes, dizen cantos leales, éstos son Agustino, Gregorio, otros tales, cuantos que escrivieron los sos fechos reales.

Les oiseaux qui chantent dans ces arbres fruitiers, qui ont de douces voix et entonnent des chants loyaux, ce sont Augustin, Grégoire, et d'autres encore, tous ceux qui écrivirent ses hauts faits royaux.

Estos avién con Ella amor e atenencia, en laudar los sos fechos metién toda femencia ; todos fablavan d'Ella, cascuno su sentencia ; pero tenién por todo todos una creencia. 
Ils avaient envers Elle amour et fidélité, à louer ses hauts faits ils consacraient tous leurs efforts tous parlaient d'Elle, chacun par sa propre sentence ; mais tous avaient en tout une seule croyance (26-27).

Cette polyphonie des chants d'oiseaux est aussi une belle façon de dire que si les voix sont multiples, elles n'en construisent pas moins un rapport à la vérité qui est unique. Et comme critère de cette vérité, se révèle une autorité que Berceo a su faire sienne. 